\title{
Adaptation Control Parameters of Multipath Model of an Adaptive Acoustic Echo Canceller
}

\author{
Sanjeev Kumar Dhull \\ Asst.Professor \\ Department of Electronics and \\ communication Engineering, Guru \\ Jambheshwar University of Science \\ and Technology, Hisar, India
}

\author{
Dr.Manoj Duhan \\ Reader\&Chairman \\ Department of Electronics and \\ communication Engineering, \\ DCRUSTM,Murthal,sonipat,india \\ india
}

\begin{abstract}
An overview of acoustic echo cancellation systems is presented. First the basics of echo's, type of echo's , why echo canceller are required and what are the difference between echo canceller are explained then the application where they can be used is discussed.. This paper presents applications of acoustic echo control and shows most recent solutions. For some selected applications (LEM) results achieved are presented.
\end{abstract}

\section{Categories and Subject Descriptors}

C.3[Special-purpose and application-based systems]: Signal processing systems ; C.4 [Performance of systems]: Design studies ,Performance attributes .

\section{General Terms}

Algorithms, Measurement, Performance, Design, Experimentation, Theory.

\section{Keywords}

Adaptive, Echo, LMS, Filter, Step size. Echo cancellation, Adaptive filters

\section{INTRODUCTION}

The rapid growth of technology in recent decades has changed the whole dimension of communications. Now in the twenty first century Echo cancellation [1] is a widespread technology in communication systems. Acoustic echo cancellers are different in nature from those for line or data echo cancellers.

The acoustic environment is wide open and changed continuously as compared to that in the transmission line and solution required are highly complex than line echo cancellers. Factors which affect or cause the disturbance in acoustic environment are Reflections, near end speech and Noise .Reflections from the walls produces [2] long impulse response for the echo path. Highly non stationary back ground noise continuously changes the degree of disturbance in coefficient adaptation of the adaptive filter. Existence of the near-end speech is also another factor for interference in coefficient adaptation. Thus the solution to these problems is the elimination of the echo.

\section{ECHO CANCELLATION}

Elimination of echo is an important and difficult task especially in case of acoustic environment. The reduction of acoustic echoes is achieved by three different approaches: The technique which was used in earlier stages was echo suppression. Echo suppression[3] is performed by means of either frequency selective or broadband attenuation. To keep the background noise at a constant level Echo suppression technique is generally combined with the injection comfort noise. due to some disadvantages of echo suppression echo cancellation came into picture $\square$ the process of Acoustic echo cancellation[7][9] is achieved with the help of adaptive filter[7] which models the loudspeaker enclosuremicrophone(LEM) system.. In case of stereo or multi-channel systems the correlation between the excitation signals needs to be reduced by utilizing either nonlinear or time-varying decorrelation methods. $\square$ In some applications such as hearing aids or carinterior communication systems the adaptation of the echo cancellation filter is rather difficult because of strong correlation between the excitation signal and the local signals. In such applications it is beneficial to exploit the spatial separation of the loudspeaker(s) and the local speaker by means of microphone arrays and beamforming. In recent systems these three approaches are often combined [3]. For the implementation different structures such as low delay broadband or computationally efficient sub band or frequency domain processing can be utilized. The latter two structures have the drawback of introducing a delay into the signal path. The most suitable processing structure[4] depends crucially on the boundary conditions of the specific application.

\section{Design Parameters}

There are a various factors which affect performance of echo canceller and should be kept in mind while doing echo cancellation[5]. These factors are, no of channel, type of filters structure, convergence speed, noise immunity, processing delay and residual echo level after convergence. Here is a brief review of all these factors. No. of taps should be sufficiently large to cover a long impulse response of the echo path in an Acoustic environment. Signal bandwidth also plays an important role in deciding the number of taps. Algorithms[6] which are used in echo cancellers are RLS and LMS algorithms and their different variants.IIR and FIR are the basic structure of filter design of echo cancellation. FIR filter is considered better for implementation[7] because of good stability and better computational capabilities The Convergence speed can be improved by fast convergence 
algorithms such as adaptive step size algorithms.[8] Coefficient adaptation should be disabled during double-talk periods because it may cause degradation in echo cancellation because of the interference by the near-end speech signals. Deciding the number of channels is also an important parameter in acoustic echo cancellation. With the growing demand of efficiency and due to advancement in the ways of communication it is the time of multichannel conference systems and multichannel acoustic echo cancellers. Echo cancellation performance is measure in term of ERL(echo return loss )and ERLE (echo return loss enhancement). ERLE is the most common performance measures for examining the' performance of the echo canceller The ERL is the ratio between the power of the returned echo and the power of the signal sent to the loudspeaker. It is measured in $\mathrm{dB}$ while ERLE is defined by the ratio between the power of the residual echo and the power of the echo as

$$
E R L=-10 \log _{10}\left(\frac{\sigma_{d}^{2}}{\sigma_{x}^{2}}\right)=-10 \log _{10}\left(\frac{E\left[d(n)^{2}\right]}{E\left[x(n)^{2}\right]}\right)
$$

Here $\sigma_{\mathrm{e}}{ }^{2}$ and ${\sigma_{\mathrm{d}}}^{2}$ are the powers of the echo signal and the input signal respectively.

ERLE is defined by the ratio between the power of the residual echo and the power of the echo as

$$
E R L E=-10 \log _{10}\left(\frac{\sigma_{e}^{2}}{\sigma_{d}^{2}}\right)=-10 \log _{10}\left(\frac{E\left[e(n)^{2}\right]}{E\left[d(n)^{2}\right]}\right)
$$

Here $\sigma_{\mathrm{e}}{ }^{2}$ is the power of the residual signal and $\sigma_{\mathrm{d}}{ }^{2}$ is the power of the microphone signal.

Now we will talk about the algorithms which we can use for implementation of AEC.LMS\&RLS are two basic algorithms for this purpose.LMS has their variants also named as PLMS and NLMS[11]. LMS algorithm based upon steepest descent method which is shown below Instead of going the direct path from the starting point to the optimum, it is easier to follow the gradient of the error function which leads to the optimum iteratively. The gradient as shown in Figure 1, is a vector pointing in the steepest uphill direction on the error surface at a given point of $w(k)$. The filter coefficient is updated by taking a step opposite the gradient direction. It goes locally "downhill" in the steepest direction to approach the optimum .The LMS[6][11][13] and their different variants can be driven using the following functions Let us define an error signal $e(n+1)$ at time $n+1$ as $e(n+1)=y(n+1)-\hat{y}(n+1)$

here $y(n+1)=h^{T} t x(n+1)$ is the output of a system and $h_{t}=\left[\begin{array}{llll}h_{t, 0} & h_{t, 1} & \ldots . . & h_{t 1 L-1}\end{array}\right]^{T}$ are response of system and $\hat{y}(n+1)=h^{T}(n) x(n+1)$ is the model filter output and $h(n)=\left[h_{0}(n) h_{1(n)} \cdots . . h_{L-1}(n)\right]^{T}$ is the model filter. One easy way to find adaptive algorithms that adjust the new weight vector $\mathbf{h}(n+1)$ from the old one $\mathbf{h}(n)$ is to minimize the following function $\mathrm{J}[\mathrm{h}(\mathrm{n}+1)]=\mathrm{d}[\mathrm{h}(\mathrm{n}+1), h(n)]+\eta \varepsilon^{2}(n+1)$

$\varepsilon(\mathrm{n}+1)=\mathrm{y}(\mathrm{n}+1)-h^{T}(n+1) x(n+1)$
Here value of $\eta$ plays an important role in updating the coefficients values. if $\eta$. is very small that the algorithm makes very small updates. On the other hand, if $\eta$. is very large, the minimization of $J[\mathbf{h}(n+1)]$ is almost equivalent to minimizing $d[\mathbf{h}(n+1)$, Hence, the different weight coefficients $h l(n+1), l=0,1$, $\ldots, L-1$, are found by solving the following equations:

$\frac{\partial d[h(n+1), h(n)]}{\partial h_{1}(n+1)}-2 \eta x(n+1-1) \varepsilon(n+1)=0$

if the new weight vector $\mathbf{h}(n+1)$ is close to the old weight vector $\mathbf{h}(n)$, replacing the a posteriori error signal with the a priori error signal $e(n+1)$ is a reasonable approximation and equation. $\frac{\partial d[h(n+1), h(n)]}{\partial h_{1}(n+1)}-2 \eta x(n+1-1) e(n+1)=0$ is much easier to solve for all distance measures d. The LMS algorithm is easily obtained from above equation by using the squared euclidean distance

$d \varepsilon[h(n+1), h(n)]=\amalg h(n+1)-h(n) \amalg_{2}^{2}$.

Using these equation and doing different mathematical operations we can find out different variants of the algorithm.

\section{Result of Multipath Model Acoustic Echo Canceller}

Following are result of a multipath model of acoustic canceller using LMS adaptive filter.Fig. 1 shows near end speech signal and Fig.2 is far end speech signal.Fig3 clearly indicates that the signals has been distorted due to echo effect and we required to get out desired signal at the Output side. The next two outcomes of multipath model clearly indicating the adaptation of filter coefficients and removal of echo's. As we know ERLE is the ratio between the power of the residual echo and the power of the echo at the input side of adaptive filter therefore the magnitude graph has lot of variation in strength according to echo signal strength.For getting results choosing the correct parameters is an important task otherwise we cannot achieve the desired target of echo cancellation.

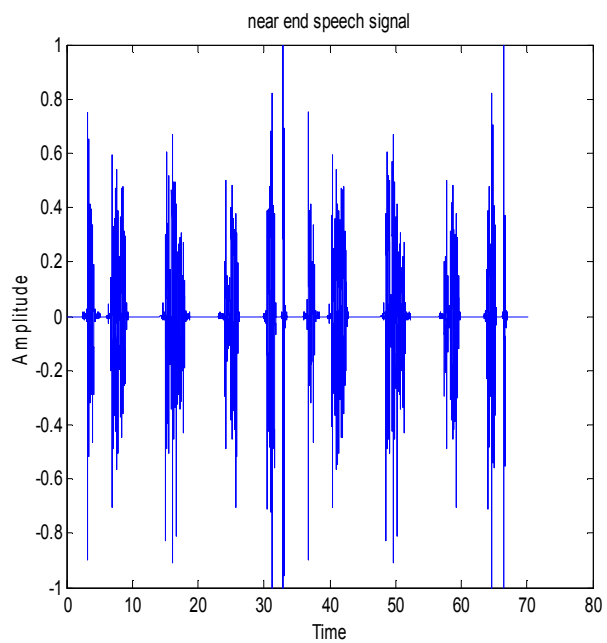

Fig1 


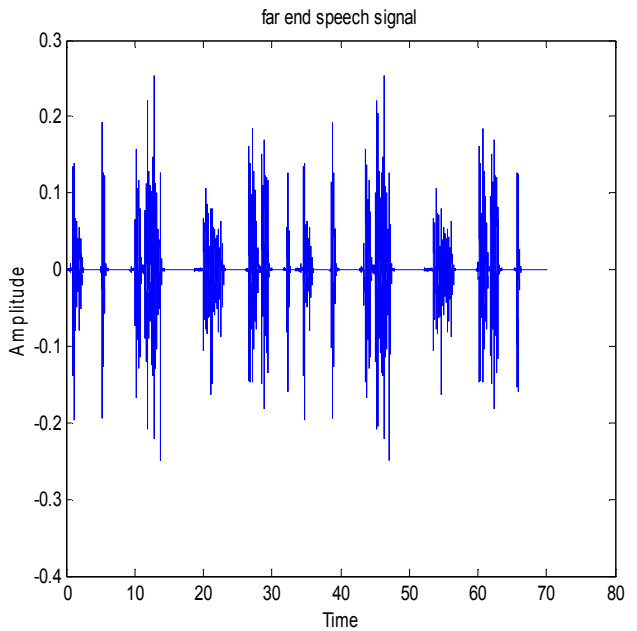

Fig.2

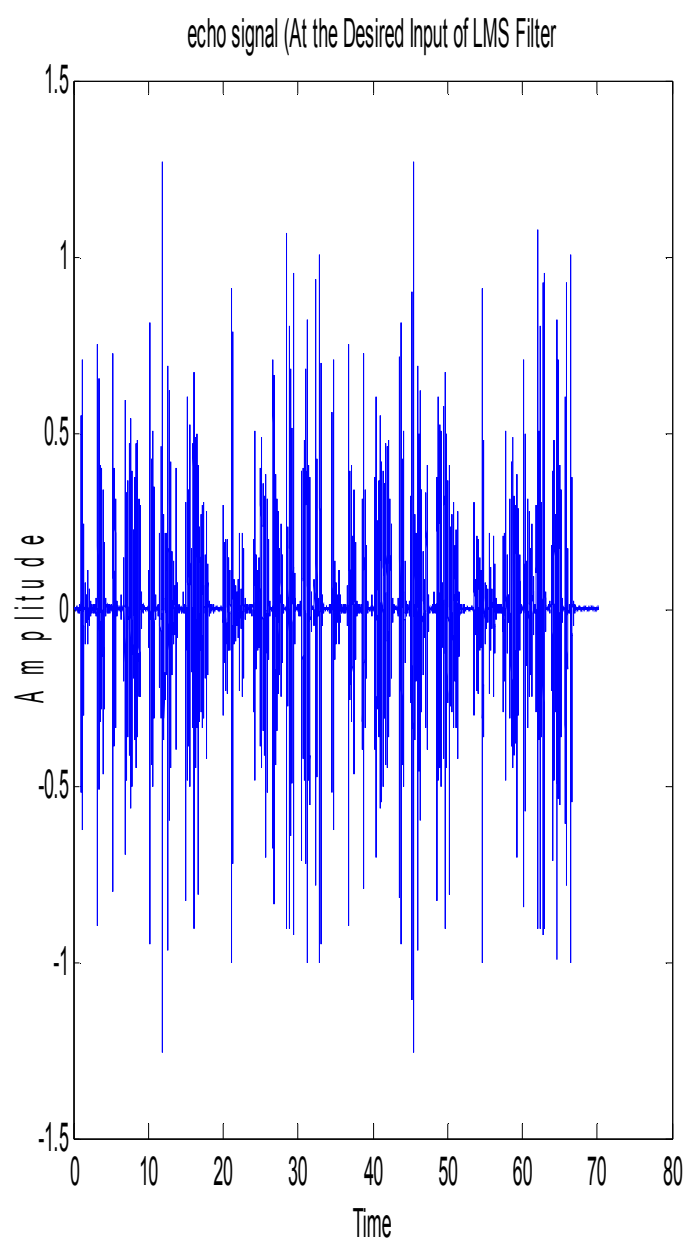

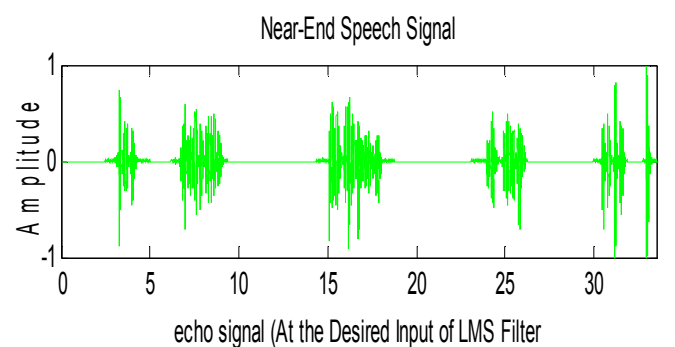
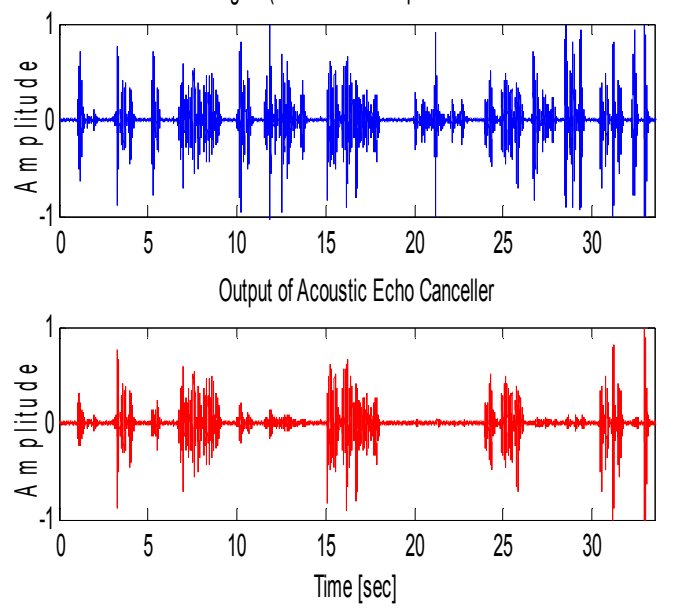

Fig.4
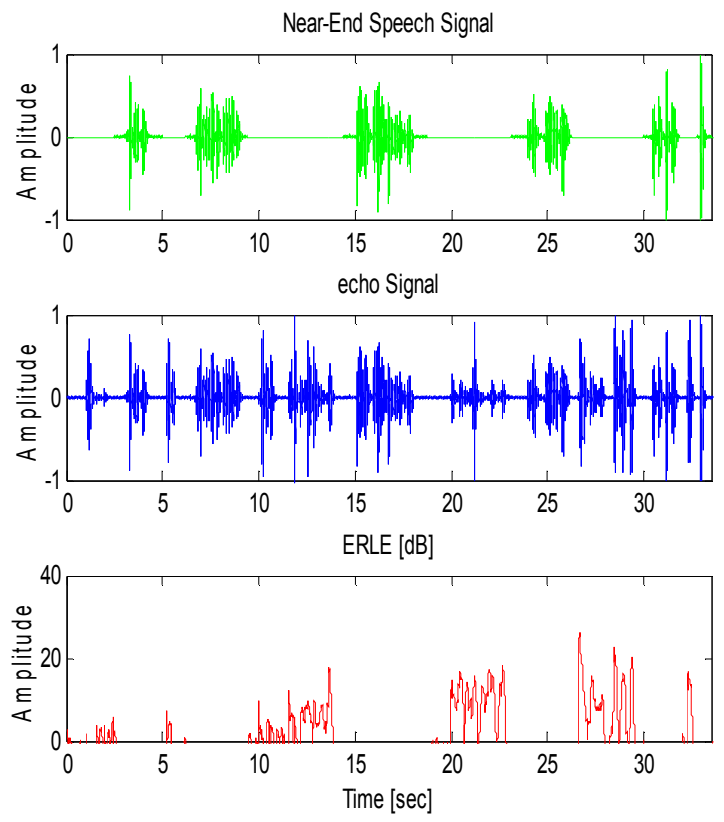

Fig.5

Fig.3 


\section{REFERENCES}

[1] S. Haykin, Adaptive Filter Theory, Englewood Cliffs, N.J., Prentice-Hall, 1991.

[2] Sondhi, M. M., and Berkley, D.A. Silencing Echoes on the Telephone Network, Proc. IEEE, Vol. 68, NO. 8, August 1980, pp. 948-963.

[3] Sondhi, M. $\boldsymbol{M}$ and Mitra, D. New Results on the Performance of a Well-Known Class of Adaptive filter, IEEE, 1976, Vol. 64, No. 11, pp. 1583-1597

[4] H. Brehm and W. Stammler, Description and generation spherically invariant speech-model signals, Signal processin vol. 12, no. 2, pp. 119-141, March 1987.

[5]M. Montazeri and P. Duhamel, A set of algorithms linking NLMS and block RLS algorithms, IEEE Signal Processing, vol. 43, pp. 444-453, Feb. 1995.

[6] T. K. Moon and W. C. Stirling, Mathematical Methods and Algorithms for Signal Processing, New Jersey: Prentice Hall, 2000 .

[7] B. Farhang-Boronjeny, Adaptive Filters Theory and Application,New York: Wiley, 2000.
[8] B. Widrow and S.D. Stearns. Adaptive Signal Processing Engleaood Cliffs, NJ: Prentict. Hall ,2001.

[9] A. Mader, H. Puder, and G. U. Schmidt, Step-size control for acoustic cancellation filters Signal Process., vol. 80, pp. 16971719, 2000[

[10] S. Ben Jebara, H. Besbes, A variable step size filtered sign algorithm for acoustic echo cancellation, IEE vol. 39, no. 12, pp. 936-938, June 2003

[11] S. Douglas, "A family of normalized LMS algorithms, IEEE signal processing letters, vol. 1, no. 3, pp. 49-51, March 1994.

[12] Sophocles J. Orfanidis, Optimum Signal processing an Introduction, McGraw hill 1988.

[13] J.G. Proakis and D.G. Manolakis, Digital Signal Processing Principles,Algorithms and Applications, Prentice Hall,1996.

[14] R. Brennan, and T. Schneider, A flexible filterbank structure for extensive signal manipulation in digital hearing aids,Proc.IEEE Int. Symp. Circuits and Systems, pp. 569-572, 1998 